\title{
Levantamento de dípteros de interesse forense em uma área de cerrado em Brasília
}

\author{
Rodrigo César da Silva Castro ${ }^{1}$ \\ Danilo Xavier Dias ${ }^{2}$ \\ Edison Ryoiti Sujii ${ }^{3}$ \\ Paulo Roberto Queiroz ${ }^{4}$
}

\section{Resumo}

A entomologia forense é a ciência que utiliza os conhecimentos sobre os insetos como ferramenta para solucionar casos em vários países. O presente estudo consistiu em analisar a frequência de coleta de famílias de dípteros de interesse forense em um fragmento de Cerrado. Foi utilizado um sistema de armadilhas suspensas a 1,5 m de altura do solo feito a partir de garrafas plásticas recicláveis, contendo isca de carne bovina em decomposição durante 26 dias. Após 60 dias de coletas, indivíduos das famílias Calliphoridae, Sarcophagidae, Muscidae e Uliididae foram identificados e a frequência de ocorrência dessas famílias foi estabelecida. Observou-se maior frequência de coleta de indivíduos das famílias Calliphoridae e Muscidae durante os primeiros 26 dias de experimento. Após esse período, as famílias Sarcophagidae e Uliididae começaram a aparecer em maior número. Dessa forma, foi possível estabelecer uma frequência de ocorrência de quatro famílias de dípteros em função de uma maior atratividade da isca em decomposição em um fragmento de Cerrado de Brasília.

Palavras-chave: Dipterofauna. Calliphoridae. Sarcophagidae. Decomposição.

Graduado em Biologia - UniCEUB;

2 Graduado em Biologia - UniCEUB;

3 PhD Ecologia. Embrapa Recursos Genéticos e Biotecnologia; e-mail: sujii@cenargen.embrapa.br

4 PhD Biologia Animal. Universidade de Brasília - UnB. Professor do curso de Biomedicina. UniCEUB; e-mail: pqsilva@uol.com.br 


\section{Introdução}

Um dos vários ramos da Biologia que vem contribuindo com as investigações criminais é a Entomologia (PUJOL-LUZ et al., 2008). Os insetos podem ser usados nas investigações de crimes de tráfico de substâncias ilícitas para determinar a possível origem delas, nos crimes de maus tratos (BENECKE, 2001; MAGAÑA, 2001; COSTA, 2003) ou, ainda, para a determinação do intervalo post-mortem (IPM) ou cronotanatognose, pois esses insetos colonizam a matéria orgânica em decomposição, indicando assim o tempo de decomposição de cadáveres (GOMES; VON ZUBEN, 2004b).

Uma das mais importantes colaborações da utilização dos insetos como ferramenta forense é a determinação do intervalo pós-morte (IPM) (COSTA, 2003; MIRANDA et al., 2006). Cada fase da decomposição cadavérica oferece condições e características próprias que atraem um determinado grupo de insetos, desencadeando um processo de sucessão heterotrófica que, se constante, pode ser altamente informativo, para o cálculo do IPM (CATTS; GOFF, 1992).

A ordem de maior importância para a entomologia forense é a Diptera (COSTA, 2003) e as famílias mais importantes são Calliphoridae, Sarcophagidae e Muscidae. A família Calliphoridae é conhecida como colonizadora inicial na fauna de sucessão cadavérica (SMITH, 1986). Dentro da família Calliphoridae, a espécie Chrysomya albiceps tem importância forense devido sua especialização em ovipositar apenas em material orgânico de origem animal (ESTRADA et al., 2009).

Os dípteros muscoides no Brasil, geralmente pertencem às famílias Muscidae, Anthomyiidae e Faniidae. Essas moscas são atraídas por dejetos humanos e matéria orgânica de origem animal e vegetal. O número de 38 espécies de Muscidae foi relacionado em associação com a atividade humana no Brasil (CARVALHO et al., 2002).

A fauna neotropical contém mais de 750 diferentes espécies de outra importante família de interesse forense, a Sarcophagidae. A maior parte das espécies dessa família é ovovivípara, depositando larvas de primeiro ínstar que iniciam imediatamente sua alimentação, diferentemente dos Calliphoridae que necessitam de mais tempo para a eclosão dos ovos (BARROS et al., 2008). 
Devido à grande importância da entomologia forense, é urgente que haja o desenvolvimento de conhecimentos, tais como, diversidade, frequência das espécies em diferentes épocas do ano e aspectos detalhados da biologia das espécies necrófagas para que possam ser utilizadas para revelar a causa, o modo, as localizações e até o tempo de morte de um indivíduo (MIRANDA et al., 2006; MISE; ALMEIDA; MOURA, 2007).

Além disso, há a necessidade de um maior conhecimento da Dipterofauna do cerrado, pois, apesar do grande número de trabalhos que são realizados na região, existe uma carência de trabalhos envolvendo Diptera (CARVALHO, 2006).

Este trabalho teve como objetivos: averiguar a incidência de Diptera de interesse forense em um fragmento de Cerrado, testar a eficiência de um sistema de captura de Díptera usando matéria orgânica em decomposição e identificar os indivíduos de interesse forense coletados nesse sistema.

\section{Material e métodos}

As coletas de Diptera foram realizadas por meio da instalação de armadilhas aéreas, segundo adaptações de Ferreira $(1978,1983)$. As armadilhas foram confeccionadas a partir de garrafas plásticas recicláveis de polietileno (PET) e constituídas por duas partes, uma inferior que possuía aberturas para a entrada das moscas e outra superior onde as moscas ficavam contidas.

Para atrair as moscas para a armadilha, utilizou-se carne bovina moída que foi mantida em um frasco fechado e deixada à temperatura ambiente durante o período de 60 dias de realização do experimento. Em intervalos de 24h, 80 g desse material em decomposição foram tirados do frasco e usados como isca na armadilha.

As coletas foram feitas em intervalos de $24 \mathrm{~h}$, sendo estas retiradas e acondicionadas individualmente em sacos plásticos para evitar a perda das amostras. Os sacos contendo as armadilhas foram mantidos a $-20^{\circ} \mathrm{C}$ e, após quinze minutos, 
os indivíduos coletados foram acondicionados em potes plásticos, contendo álcool 70\%, devidamente identificados e individualizados para cada armadilha.

Visando evitar qualquer tipo de interferência da isca anterior, as armadilhas foram lavadas diariamente com uma solução de hipoclorito 10\%, água e etanol $70 \%$, antes de se colocar nova isca.

Em seguida, as amostras foram separadas, classificadas e contadas em função das famílias e espécies de Díptera com a ajuda de uma lupa estereoscópica. Os indivíduos foram separados por dia de coleta, respeitando-se as amostras de cada armadilha e classificados ao nível de família, usando a chave de identificação de Carvalho e Mello-Patiu (2008).

As coletas foram realizadas em um fragmento remanescente de cerrado localizado em uma área urbana de Brasília. A coleta teve início no dia 11 de setembro de 2008 e foi finalizada no dia 11 de novembro do mesmo ano. A área apresentava vegetação típica de cerrado e os pontos selecionados foram dispostos, distantes de possíveis ações antrópicas. Três pontos foram escolhidos aleatoriamente, julgando-se apenas a altura de $180 \mathrm{~cm}$ necessária para a colocação das armadilhas e mantendo-se uma distância superior a $5 \mathrm{~m}$ entre elas. Foram determinados pontos específicos para cada ponto de coleta e, consequentemente, para cada armadilha. As armadilhas receberam numeração própria e cores nas tampas que serviram como forma de identificação. As cores vermelha, azul e amarela das tampas das armadilhas representaram respectivamente as armadilhas 1, 2 e 3 . As coordenadas exatas de cada armadilha também foram aferidas sendo: ponto 1: 15 46' 06.4" S - 47º 53' $45.2^{\prime \prime} \mathrm{W}$ referente à localização da armadilha 1 (tampa vermelha); ponto 2: $15^{\circ} 46^{\prime}$ $06.8^{\prime \prime} \mathrm{S}-4^{\circ} 53^{\prime} 45.5^{\prime \prime} \mathrm{W}$ referente à armadilha 2 (tampa azul); ponto 3: 15 46'06.6" $\mathrm{S}-4^{\circ} 53^{\prime} 45.9^{\prime \prime} \mathrm{W}$ referente à armadilha 3 (tampa amarela).

Foram aferidos os parâmetros climáticos com a ajuda de um termohigrômetro (Termo-Higrometro Digital Máximo e Mínimo interno/externo, Equitherm) antes e depois das coletas. 


\section{Resultados}

Ao final dos 60 dias de experimento, foram coletados 5.976 indivíduos e estes foram identificados como pertencentes às famílias Calliphoridae, Sarcophagidae, Muscidae e Uliidide.

Foram identificadas duas espécies de Calliphoridae, ou seja, C. albiceps e C. megacephala, com 3.931 e 133 indivíduos.

A família Muscidae foi a segunda família mais abundante, com 1.396 indivíduos, e a Sarcophagidae foi a quarta família mais coletada no experimento, com 120 espécimes.

Observou-se que a terceira família de Diptera mais coletada foi Uliididae, com 272 indivíduos. Além disso, os insetos resultantes de coletas acidentais corresponderam a 151 indivíduos.

A família Calliphoridae (C. albiceps e C. megacephala) representou $68 \%$ dos indivíduos coletados; Muscidae, 22,9\%; Sarcophagidae, 2\%; Uliididae, 4,6\%; e "outros" com 2,5\%. Dentro da família Calliphoridae, C. albiceps representou $65,8 \%$ e C. megacephala, 2,2\% dos indivíduos coletados, respectivamente (Tabela 1).

Em seguida, para a avaliação do comportamento das famílias, utilizando-se o sistema de coleta contendo a isca, determinou-se a distribuição temporal das famílias de Diptera ao longo do período de realização do experimento.

A família Calliphoridae correspondeu a maior parte das coletas. C. albiceps apresentou maior atividade na isca no intervalo correspondente do $12^{\circ}$ ao $29^{\circ}$ dias de decomposição e C. megacephala com maior período de atividade entre o $26^{\circ}$ e $40^{\circ}$ dias (Figura 1). 
Tabela 1 - Número total de indivíduos coletados ao longo de 60 dias de coleta ,utilizando-se isca de carne bovina em decomposição.

\begin{tabular}{|c|c|c|c|c|c|c|}
\hline \multirow{2}{*}{$\begin{array}{l}\text { Dia de } \\
\text { coleta }\end{array}$} & \multicolumn{2}{|c|}{ Calliphoridae } & \multirow{2}{*}{ Muscidae } & \multirow{2}{*}{ Sarcophagidae } & \multirow{2}{*}{ Uliididae } & \multirow{2}{*}{ Outros } \\
\hline & C. albiceps & C. megacephala & & & & \\
\hline 1 & 0 & 0 & 0 & 1 & 0 & 1 \\
\hline 5 & 292 & 12 & 264 & 25 & 14 & 2 \\
\hline 8 & 320 & 5 & 143 & 15 & 22 & 17 \\
\hline 12 & 257 & 1 & 315 & 14 & 20 & 14 \\
\hline 26 & 1529 & 26 & 306 & 5 & 59 & 27 \\
\hline 29 & 622 & 30 & 233 & 6 & 22 & 5 \\
\hline 40 & 416 & 31 & 53 & 13 & 48 & 3 \\
\hline 50 & 58 & 6 & 30 & 8 & 15 & 23 \\
\hline 54 & 383 & 13 & 12 & 7 & 22 & 9 \\
\hline 62 & 54 & 9 & 13 & 26 & 50 & 50 \\
\hline Total & 3.931 & 133 & 1.369 & 120 & 272 & 151 \\
\hline $\begin{array}{l}\text { Total } \\
\text { geral }\end{array}$ & & & 5.9 & & & \\
\hline
\end{tabular}

Figura 1 - Número total de indivíduos das espécies C. albiceps (A) e C. megacephala (B) ao longo de 60 dias de coleta, utilizando-se um sistema de captura contendo isca bovina em decomposição.
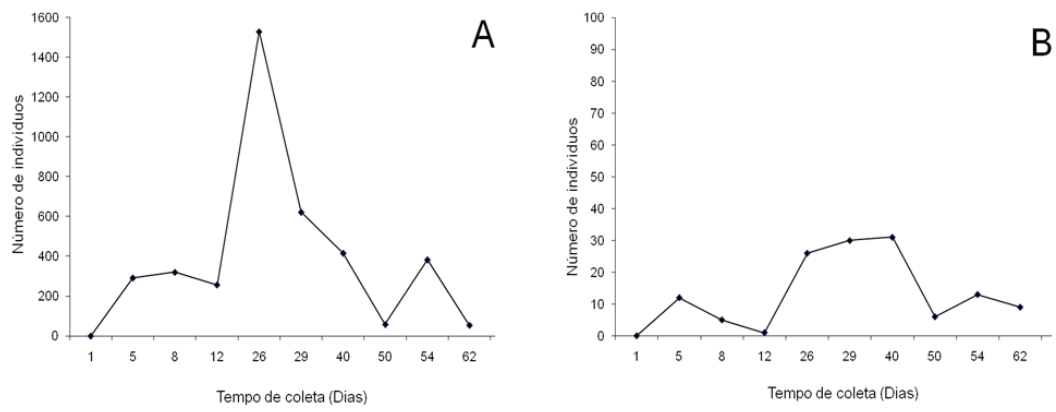
Observou-se que o pico de coleta para C. albiceps correspondeu ao $26^{\circ}$ dia de decomposição da isca e, para a espécie C. megacephala, correspondeu ao intervalo do $26^{\circ}$ ao $40^{\circ}$ dia de experimento.

As curvas de distribuição do número total de indivíduos coletados da família Muscidae apresentaram diferença em relação aos indivíduos da família Sarcophagidae (Figura 2).

Figura 2 - Número total de indivíduos das famílias Muscidae (A) e Sarcophagidae (B) coletados ao longo de 60 dias de coleta em sistema de captura contendo isca bovina em decomposição.
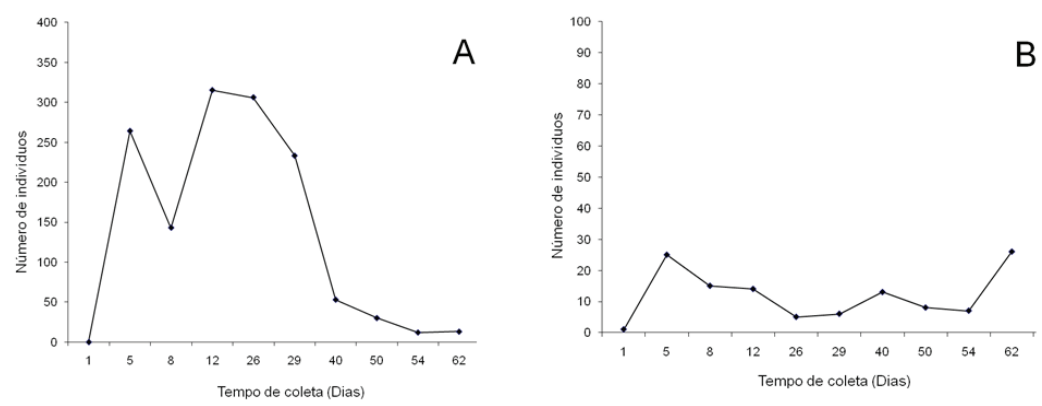

A família Muscidae representou a segunda maior família de Diptera capturada. O pico de atividade dessa família correspondeu ao intervalo do $12^{\circ}$ ao $29^{\circ}$ dias de coleta. Sarcophagide foi a quarta família mais coletada, não observando-se pico expressivo ao longo do período de realização do experimento.

A família Uliididae, constituída por indivíduos de porte pequeno, apareceu em maior número em relação à família Sarcophagidae nesse experimento (Figura 3). Os indivíduos da família Uliididae tiveram dois picos de captura, no $26^{\circ}$ e $40^{\circ}$ dias de coleta. 
Figura 3 - Número total de indivíduos da família Uliididae coletados ao longo do período de 60 dias de realização do experimento.

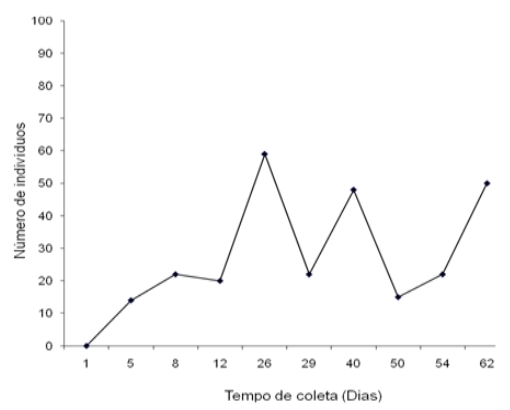

Os indivíduos foram agrupados nas suas famílias, e determinada a porcentagem de coleta a cada dia. A partir dessa informação, foi elaborado um gráfico para avaliar a distribuição das famílias de Diptera ao longo do intervalo de realização do experimento (Figura 4).

Figura 4 - Distribuição das três principais famílias de Diptera ao longo dos 60 dias de realização do experimento.

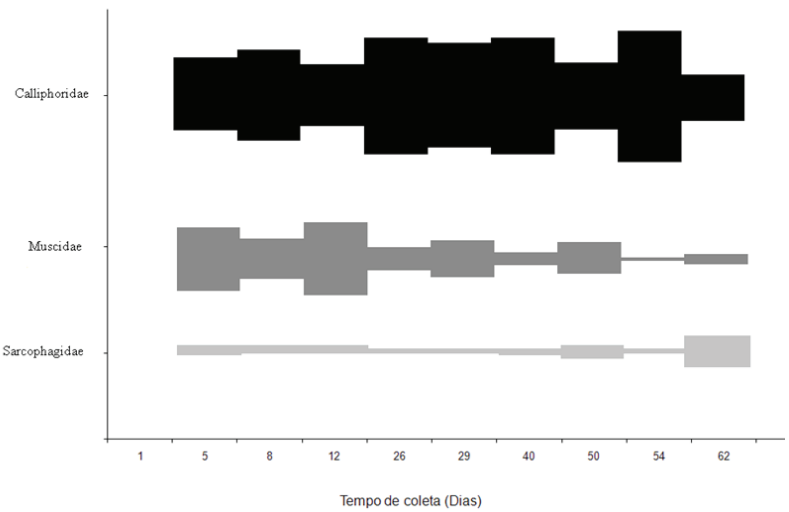

Observou-se que Calliphoridae foi a família de Diptera dominante no estudo, apresentando pico de atividade no período entre o $12^{\circ}$ e $40^{\circ}$ dias de coleta. Além disso, essa família apresentou comportamento de visita à matéria orgânica em decomposição, durante todo o período de estudo. 
Para Muscidae, observou-se maior frequência de visita até o $12^{\circ}$ de realização do experimento, havendo redução até o término do estudo. Sarcophagidae visitou a isca por todo o período de realização do experimento. Contudo, em termos numéricos, foi a família menos abundante nas coletas. $\mathrm{O}$ aumento na atividade dos indivíduos ocorreu após o $55^{\circ}$ dia de experimento.

Para analisar possíveis variáveis que poderiam influenciar na frequencia de visitas de Diptera, a temperatura foi aferida em cada ponto de coleta, obtendo-se uma média de temperatura local do estudo. Os dados de umidade foram obtidos no Instituto Nacional de Meteorologia (INMET). Compararam-se, então, os dados de temperatura e umidade ao longo do intervalo de tempo de realização do experimento (Figura 5).

Figura 5 - Variação da temperatura e a da umidade durante os dias de realização do experimento.

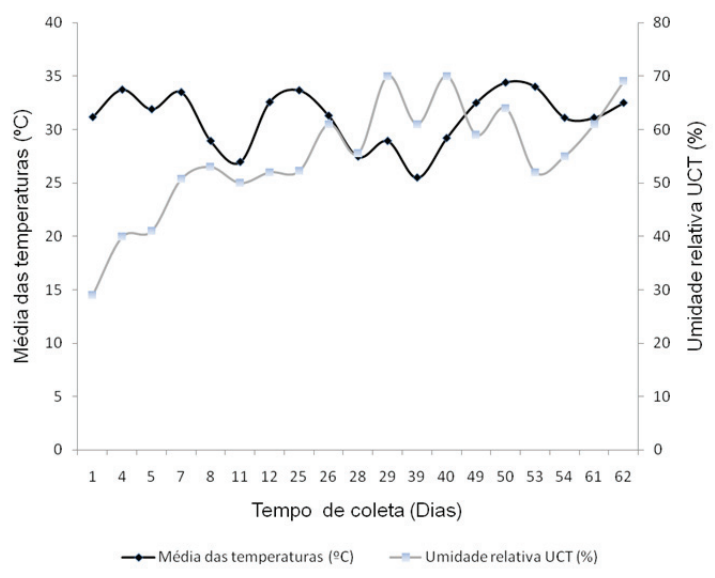

A temperatura e a umidade não exerceram influência sobre o comportamento de Diptera, uma vez que não houve relação entre os picos de coleta de cada família e as variações de temperatura e umidade. Por meio da análise dos gráficos, foi possível observar que as coletas mantiveram-se contínuas, apresentando um nível de coleta maior ou menor de uma espécie relacionada com a fase de decomposição em que se encontrava a isca. A maior influência no experimento foi a incidência ou não de chuva. Quando ocorria chuva, o número de indivíduos coletados era baixo. 


\section{Discussão}

A região onde as armadilhas foram colocadas influenciou decisivamente na quantidade das espécies que foram coletadas, confirmando que o habitat, a vegetação, o solo e a pluviosidade têm impacto nos tipos e espécies de insetos presentes (ESTRADA et al., 2009).

As comunidades também variam com o tempo, ou seja, determinadas espécies que colonizam a matéria orgânica em um determinado estado de decomposição podem depender desse tempo. Os artrópodes também podem ser afetados por dinâmicas sazonais. Algumas espécies podem aparecer em uma estação e em outra não (ARNALDOS et al., 2004).

O local de coleta era um fragmento de cerrado, consideravelmente afastado de edificações e ruas, simulando um ambiente livre de ação antrópica. Existe uma carência de informações de ocorrência de Diptera no cerrado do Planalto Central, sobretudo os insetos necrófagos de interesse forense (SANTANA, 2006).

Observou-se que a ordem Diptera correspondeu a 98,09\% dos indivíduos coletados. De acordo com Benecke (2005), centenas de espécies de artrópodes são atraídas por corpos em decomposição, primeiramente, moscas (Diptera), besouros (Coleoptera) e suas larvas. Carvalho et al. (2000) ao estudarem os insetos que visitam corpos de Sus scrofa em uma área natural de floresta e corpos humanos no IML em São Paulo, também encontraram um pico de atividade de dípteros nas fases iniciais de decomposição e coleópteros nos estágios finais.

Quanto ao sistema de armadilha, o uso de carcaça de porco como isca, por se assemelhar ao processo de decomposição de carne humana, é um dos mais utilizados com variações na forma da armadilha onde esta é utilizada. Archer e Elgar (2003) utilizaram latas de lixo de 50 litros como armadilha e carcaças de filhotes de porco como isca. Carvalho et al. (2000) utilizaram 16 carcaças de porco como isca para atrair moscas em uma floresta em São Paulo. Esses animais ficavam presos dentro de gaiolas de metal para evitar a predação por outros animais. Para esse tipo de modelo, nos vários estágios de decomposição, tem destaque a invasão das famílias Calliphoridae, Muscidae e Sarcophagidae (MISE et al., 2007). Porém, 
muitos estudos de atração de moscas para o corpo são realizados utilizando-se pedaços de carne e outros tipos de isca (ARCHER; ELGAR, 2003). Gomes e Von Zuben (2004b) utilizaram uma carcaça de peixe para capturar adultos de L. cuprina em um experimento na Universidade Estadual Júlio Mesquita Filho (UNESP). Carreira et al. (2008) usaram um sistema de armadilhas confeccionadas a partir de garrafas do tipo PET e utilizaram $100 \mathrm{~g}$ de carne bovina como isca, que não foi substituída durante 30 dias de experimento em uma área localizada no Lago Norte, Brasília. O mesmo sistema de captura utilizando garrafas PET (armadilhas) e carne como isca foi utilizado neste trabalho e permitiu a coleta de um grande número de dípteros não oferecendo dificuldades para a coleta dos indivíduos.

Quando há perda de partes, identificar as espécies, muitas vezes, é impossível por métodos taxonômicos tradicionais (CARREIRA et al., 2008). Assim, um ponto importante a ser destacado foi a estrutura da armadilha, pois esta contribuiu para a captura dos indivíduos sem grandes perdas de estruturas anatômicas deles. Isso colaborou para uma melhor análise durante a triagem e identificação de Diptera pela chave dicotômica.

Durante o experimento, foi observada a captura de um número superior de indivíduos da família Calliphoridae. Espécies dessa família estão entre as mais utilizadas em experimentos envolvendo entomologia forense. São importantes, pois podem apresentar distribuição temporal e espacial extremamente específica (CARREIRA et al., 2008). A grande quantidade da família Calliphoridae registrada pode ter uma explicação comportamental: essa espécie deposita seus ovos no corpo após poucas horas da morte (LANNACONE, 2003). O gênero Chrysomya foi o mais coletado. Em estudo realizado em um pomar, na circunscrição de Goiânia, foram coletados 3.154 indivíduos do gênero Chrysomya, sendo 62,62\% destes $C$. albiceps (FERREIRA; SANTOS; CUNHA, 1995). Santana (2006) realizou coletas de dípteros em uma área rural do Distrito Federal. A captura total de califorídeos foi $38 \%$ do total coletado. C. albiceps representou 95,08\% dos indivíduos da família Calliphoridae coletados.

Em relação à família Muscidae, observa-se outro tipo de comportamento por serem espécies que se adaptam facilmente ao ambiente onde estão e, no ambiente 
urbano, detêm um número superior de espécimes (D’ALMEIDA; ALMEIDA, 1998). Algumas espécies se deslocam para habitações e agem como potenciais vetores de patógenos (CARVALHO et al., 2002). Neste estudo, representaram $22,9 \%$ das capturas, e assim, foi a segunda família mais coletada no experimento. Entretanto, Carreira et al. (2008), em estudo feito no Lago Norte-DF, em uma área tipicamente urbana, coletaram 406 (36,6\%) indivíduos dessa família de um total de 1.116 moscas capturadas.

Com menor ocorrência em relação às outras famílias, porém não menos importante, foram coletados indivíduos da família Sarcophagidae. Em tese, essa família tem como característica a preferência por estágios mais avançados de decomposição do cadáver para a reprodução (SOUZA, 1994). Entretanto, Barros et al. (2008) utilizando uma carcaça de porco, em uma área de cerrado, definiram essa família como pioneira. Ainda, Sarcophagidae apresenta grande importância em estudos sobre a fauna cadavérica em áreas de Cerrado, sobretudo, no Distrito Federal. Foram coletados 120 indivíduos de Sarcophagidae durante todo o experimento ( $2 \%$ do total coletado). Apesar de eles aparecerem com menor frequência, foram constantemente capturados desde o início do experimento. Observou-se que Sarcophagidae, apesar de aparecer em número reduzido, esteve presente em quase todas as coletas. Portanto, neste trabalho, a ocorrência das três famílias foi similar a trabalhos anteriores e a isca de carne bovina foi atrativa principalmente para Calliphoridae e Muscidae, seguido de Sarcophagidae que também foi atraída pela isca todos os dias, apesar de aparecer em número reduzido.

Essas informações reforçam a necessidade de realização de experimentos com delineamentos que possam explorar mais fatores ambientais e bioquímicos que estejam associados e que influenciem no processo de decomposição da matéria orgânica.

A partir da análise dos resultados desta pesquisa, pôde-se chegar às seguintes conclusões: o sistema de coleta utilizado nesse experimento mostrou-se adequado para a coleta de Diptera das famílias Calliphoridae, Muscidae, Sarcophagidae e Uliididae; a isca, utilizando matéria orgânica em decomposição, mostrou-se adequada para a atração das famílias anteriormente citadas; as condições ambientais, 
temperatura e umidade, não exerceram influência pronunciada na frequência de ocorrência de Diptera; Calliphoridae foi a espécie de Diptera mais coletada, seguindo-se Muscidae e Sarcophagidae; as espécies C. albiceps e C. megacephala apresentaram frequências de ocorrência diferenciadas; Muscidae apresentou frequência de ocorrência diferenciada em relação a de Calliphoridae e Sarcophagidae; Sarcophagidae apresentou baixa frequência de ocorrência na matéria orgânica, mas mantendo-se presente por todo o período de realização do experimento.

O que ficou evidente pela análise dos dados foi uma maior incidência das famílias Calliphoridae e Muscidae durante os primeiros dias de experimento, com baixa captura de indivíduos das famílias Sarcophagidae e Uliididae. Dessa forma, foi possível definir um padrão de distribuição das famílias de Diptera em virtude de uma maior atratividade por um determinado período de decomposição da carne.

\section{Dipteran survey of forensic interest in an area of cerrado in Brasilia}

\section{Abstract}

Forensic entomology is the science that uses knowledge about insects as a tool to resolve cases in several countries. This study analyzed the frequency of collection of families of Diptera of forensic interest in a fragment of Cerrado. In this study it was used a system of traps suspended $1.5 \mathrm{~m}$ above the soil made from recycled plastic bottles baited with decomposing beef for 26 days. After 60 days of collection, individuals of the families Calliphoridae, Sarcophagidae, Muscidae and Uliididae were identified and the frequency of occurrence of these families has been established. There was greater frequency of collection of individuals from the families Calliphoridae and Muscidae during the first 26 days of experiment. After this period, the families Sarcophagidae and Uliididae began appearing in greater numbers. Thus, it was possible to establish a frequency of four families of flies due to an increased attractiveness of the bait on a rotting piece of Cerrado de Brasília.

Keywords: Dipterofauna. Calliphoridae. Sarcophagidae. Decomposition. 


\section{Referências}

ARCHER, M. S.; ELGAR, M. A. Effects of decomposition on carcass attendance in a guild of carrion-breeding flies. Medical and Veterinary Entomology, [S.l.], v. 17, p. 263-271, 2003.

ARNALDOS, M. I. et al. A forensic entomology case from the southeastern Iberian Peninsula. Aggrawal's Internet Journal of Forensic Medicine and Toxicology, [S.l.], v. 5, n. 1, p. 22-25, 2004.

BARROS, R. M. et al. Sarcophagidae (insecta, diptera) associados à decomposição de carcaças de sus scrofa linnaeus (suidae) em área de cerrado do Distrito Federal, Brasil. Revista Brasileira de Entomologia, Curitiba, v. 52, n. 4, p. 606-609, 2008.

BENECKE, M. A brief history of forensic entomology. Forensic Science International, [S.1.], v. 120, p. 2-14, 2001.

BENECKE, M. Arthropods and corpses. Forensic Pathology Reviews, [S.l.], v. 2, p. 207-240, 2005.

CARREIRA, G. A. et al. Levantamento e caracterização da dipterofauna necrófaga em uma localidade de Brasília. Universitas: ciências da saúde, Brasília, v. 6, n. 2, p. 87-102, 2008.

CARVALHO, C. J. B. ; MELLO-PATIU, C. A. Key to the adults of the most common forensic species of diptera in South America. Revista Brasileira de Entomologia, Curitiba, v. 52, n. 3, p. 390-406, 2008.

CARVALHO, C. J. B. et al. Chave para adultos de dípteros (muscidae, faniidae, anthomyiidae) associados ao ambiente humano no Brasil. Revista Brasileira de Entomologia, Curitiba, v. 46, n. 2, p. 107-114, 2002.

CARVALHO, L. M. L. et al. A checklist of arthropods associated with pig carrion and human corpses in Southeastern Brazil. Memórias do Instituto Oswaldo Cruz, Rio de Janeiro, v. 95, n. 1, p. 135-138, 2000.

CARVALHO, L. S. Redescrição das larvas de terceiro ínstar de cinco espécies de dípteros califorídeos (insecta, diptera) de importância para a entomologia forense. 2006. $67 \mathrm{f}$. Dissertação (Mestrado em Biologia animal)-Universidade de Brasília, Brasília, 2006.

CATTS, E. P.; GOFF, M. L. Forensic entomology in criminal investigations. Annual Review of Entomology, [S.1.], v. 37, p. 253-272, 1992. 
COSTA, J. O. Entomologia forense: quando os insetos são vestígios. São Paulo: Millenium, 2003.

D’ALMEIDA, J. M.; ALMEIDA, J. R. Nichos tróficos em dípteros caliptrados, no Rio de Janeiro. Revista Brasileira de Biologia, São Carlos, SP, v. 58, n. 4, p. 563570, 1998.

ESTRADA, D. A. et al. Taxa de desenvolvimento de chrysomya albiceps (wiedemann) (diptera: calliphoridae) em dieta artificial acrescida de tecido animal para uso forense. Neotropical Entomology, [S.1.], v. 38, n. 2, p. 203-207, 2009.

FERREIRA, M. J. M. Sinantropia de calliphoridae (diptera) em Goiânia, Goiás. Revista Brasileira de Biologia, São Carlos, SP, v. 43, p. 193-210, 1983

FERREIRA, M. J. M. Sinantropia de dípteros muscóides de Curitiba, Paraná. I. Calliphoridae. Revista Brasileira de Biologia, [S.l.], v. 38, p. 445-454, 1978.

FERREIRA, M. J. M.; SANTOS, B. B.; CUNHA, H. F. Flutuação populacional de espécies de chrysomya robineau-desvoidy (diptera, calliphoridae) em Pomar de Goiânia, Goiás, Brasil. Revista Brasileira Zoologia, São Carlos, SP, v. 12, n. 31, p. 557-562, 1995.

GOMES, L.; VON ZUBEN, C. J. Dispersão larval radial pós-alimentar em lucilia cuprina (diptera, calliphoridae): profundidade, peso e distância de enterramento para pupação. Iheringia, Série Zoologia, [S.1.], v. 94, n. 2, p. 135-138, 2004a.

GOMES, L.; VON ZUBEN, C. J. Efeito da temperatura na profundidade de enterramento de larvas de chrysomya megacephala (fabricius, 1794) (diptera: calliphoridae) sob condições controladas. Entomologia y Vectores, [S.l.], v. 11, n. 3, p. 551-557, 2004b.

LANNACONE, J. Artropofauna de importância forense en un cadaver de cerdo en el Callao, Perú. Revista Brasileira de Zoologia, Curitiba, v. 20, n. 1, p. 85-90, 2003.

MAGAÑA, C. La entomología forense y su aplicación a la medicina legal. data de la muerte. Aracnet, [S.1.], v. 28, p. 49-57, 2001.

MIRANDA, G. H. B. et al. Coleta de amostra de insetos para fins forenses. Ministério da Defesa, Departamento da Polícia Federal, Diretoria TécnicoCientífica, Instituto Nacional de Criminalística. Brasília, 2006. 
MISE, K. M.; ALMEIDA, L. M.; MOURA, M. O. Levantamento da fauna de coleoptera que habita a carcaça de sus scrofa L., Curitiba, Paraná. Revista Brasileira de Entomologia, Curitiba, v. 51, n. 3, p. 358-368, 2007.

PUJOL-LUZ, J. R.; ARANTES, L. C.; CONSTANTINO, R. Cem anos da entomologia forense no Brasil (1908-2008). Revista Brasileira de Entomologia, Curitiba, v. 52, n. 4, p. 485-492, 2008.

SANTANA, F. H. A. Dipterofauna associada a carcaças de sus scrofa linnaeus em área de cerrado do Distrito Federal, com ênfase na família calliphoridae (insecta, diptera). 2006. 92f. Dissertação (Mestrado em Biologia Animal)-Instituto de Ciências Biológicas, Universidade de Brasília, Brasil.2006.

SMITH, K. G. V. A manual of forensic entomology. Londres: The Trustees of the British Museum, 1986.

SOUZA, A. M. Sucessão entomológica na decomposição de carcaça animal, com ênfase nas famílias calliphoridae e sarcophagidae (diptera). 1994. $96 \mathrm{f}$. Dissertação (Mestrado em Parasitologia)-Universidade Estadual de Campinas, Campinas, 1994. 